\title{
Statistical accuracy analysis of different detecting algorithms for surveillance system in smart city
}

\author{
Hassan Al-Yassin ${ }^{1}$, Jaafar I. Mousa ${ }^{2}$, Mohammed A. Fadhel ${ }^{3}$, Omran Al-Shamma ${ }^{4}$, Laith Alzubaidi ${ }^{5}$ \\ $1,3,4,5$ University of Information Technology and Communications, Iraq \\ ${ }^{2}$ Israa University College, Iraq \\ ${ }^{5}$ Faculty of Science \&Engineering, Queensland University of Technology, Australia
}

\begin{tabular}{l} 
Article Info \\
\hline Article history: \\
Received Sep 23, 2019 \\
Revised Nov 25, 2019 \\
Accepted Nov 9, 2019 \\
\hline Keywords: \\
Background subtraction \\
Gaussian mixture models \\
K-way ANOVA \\
SPSS \\
Temporal differencing
\end{tabular}

\section{Corresponding Author:}

Mohammed A. Fadhel,

Business Informatics Collage,

University of Information Technology and Communications,

Baghdad, Iraq.

Email: mohammed.a.fadhel@uoitc.edu.iq

\begin{abstract}
Several detecting algorithms are developed for real-time surveillance systems in the smart cities. The most popular algorithms due to its accuracy are: Temporal Differencing, Background Subtraction, and Gaussian Mixture Models. Selecting of which algorithm is the best to be used, based on accuracy, is a good choise, but is not the best. Statistical accuracy anlysis tests are required for achieving a confident decision. This paper presents further analysis of the accuracy by employing four parameters: false recognition, unrecognized, true recognition, and total fragmentation ratios. The results proof that no algorithm is selected as the perfect or suitable for all applications based on the total fragmentation ratio, whereas both false recognition ratio and unrecognized ratio parameters have a significant impact. The mlti-way Analysis of Variate (so-called K-way ANONVA) is used for proofing the results based on SPSS statistics.
\end{abstract}

Copyright (c) 2020 Institute of Advanced Engineering and Science. All rights reserved.

\section{INTRODUCTION}

The recent decade shows an incredible progression in computers, technologies, and communications, which advances the growth of smart cities, as well as, smart automated systems [1]. Automatic video surveillance is one of such smart systems. It is widely applied in real-time traffic monitoring. Based on video cameras, the system has the ability to detect and recognize accidents, track and analyze vehicles, and traffic flow [2]. The current challenges are in developing a fully automated system, which characterizes high storage capacity in a small space, very fast processing (real-time processing), and does not need specific task processing or tuning. Thus, these challenges pointed out the need for smart algorithms with the capability of identifying and recognizing tasks on the real-time basis [3].

Conversely, detecting and tracking objects that are in apparent movement is performed by analyzing successive frames in a video surveillance using image analysis algorithms [4, 5]. More specifically, these algorithms detect pixels, which are diverse from the background, and suspect them as a percentage of a new actual object to the foreground [6]. Hence, several algorithms for real-time video surveillance are introduced. Temporal Differencing, Background Subtraction, and Gaussian Mixture Models are examples of such algorithms [7]. In particular, the best parameter used for evaluating the performance of these algorithms is the accuracy. However, this parameter is somewhat ambiguous, dependent on the training data and the testing data. Thus, achieving a more confident (accurate) parameter is through statistical tools. For instance, t-tests, general linear models, and analysis of variance, are commonly used tools. For multi-group comparison, the Analysis of Variance (so-called ANOVA) is the most popular and efficient tool. 
This paper proposes a state-of-art trail for applying the K-way ANOVA test in comparing between algorithms rather than groups. Three detecting algorithms for video surveillance, which are Temporal Differencing, Background Subtraction, and Gaussian Mixture Models, are employed with a common training and testing data for all these algorithms. The results show that the Background Subtraction algorithm has the most priority among the others.

\section{LITERATURE REVIEW}

In the recent years, several algorithms are developed for video surveillance recognition. For instance, Sehairi et al. [8] proposed a simplified self-organized Background Subtraction algorithm, while Martins et al. [9] used different methods to explain the difficulty and trouble of Background Subtraction algorithm that based on the Background model, known as Mixture of Gaussians (MoG). Henry et al. [10] introduced a multi-object detection and tracking model based on stereo vision for use in surveillance systems. Their model overcomes the object occlusion, shadow interference, and problems of illumination variation. To protect a point according to their locations on the plane and their height values, a Kernel-based Clustering algorithm is used. In addition, a reconfigurable hardware is implemented using a parallel architecture as an object tracking system, which is based on the Background Subtraction algorithm [11]. Another studies [12] [13] proposed two different object detection algorithms, namely Temporal Difference algorithm and Fixed Background algorithm for a video of (120 x 160) pixels frame-size.

On the other hand, statistics and statistical algorithms are employed widely in detection algorithm for video surveillance. Albiol, et al. [14] presented an approach, using statistical method, for inferring what is happening in a crowded scene. It is based on detecting salient points (corners) along with their motion vectors, instead of segmenting and tracking the individuals in each frame. Then, based on this data, the statistical measures are determined. Liu, et al. [15] proposed an innovative method to detect anomaly in video surveillance systems. The proposed algorithm is doing well in detecting unusual events in the video surveillance with maintaining a low false alarm rate. However, the last years show introducing some novel algorithms for video object detecting based on statistics $[16,17]$.

However, several studies discussed the theory behind and meaning of K-way ANOVA and descriptive discriminant analysis (DDA) [18]. Konietschke and Frank [19], developed parametric and nonparametric bootstrap methods for multi-factor multivariate data. Later, Engel proposed an enhanced version that is basically a weighted mean of the ASCA and multi-variate ANOVA models [20], whereas Asiltürk, et al. [21], studied modelling of surface roughness for medical alloy machined using CNC depend on cutting parameters, which are optimized by ANOVA. In the latest years, deep learning has gained more pupularty and showed great performace in several pattern recognistion tasks [22-24] and in surveillance systems as well [25].

This paper presents a statistical accuracy analysis for comparison between three detecting algorithms of video surveillance. This analysis give an evidence of the used algorithm accuracy among other algorithms.

\section{METHODOLOGY}

As mentioned earlier, three detection algorithms are employed for comparison in this paper, due to their efficient and most popular use. Whereas, K-way ANOVA technique is employed for comparison basis. It is the best technique for comparison multiple groups of data $[2,18]$.

\subsection{Definitions}

Here shown a brief definition for Temporal Differencing, Background Subtracting, and Gaussian Mixture Models detection algorithms is introduced.

\subsubsection{Temporal Differencing (TD)}

This algorithm is straightforward and uncomplicated in implementation. It is particularly convenient to dynamic scene variations. TD utilizes two consecutive (neighboring) images based on a video segment (sequence of images on a time basis) for subtracting and getting difference images. The outcome of the subtraction process obtains the information of the moving target via a threshold value. In contrast, one drawback of this algorithm is unsuccessful in detecting all significant pixels of a few kinds of moving objects. Another drawback is its need for specialized hardware in order to successfully detection of the unmoved objects in real-time basis. 


\subsubsection{Background Subtraction}

It is commonly used for object detection in stationary background images [26, 27]. The first step in this algorithm is generating a reference background image through averaging images over time. Next, it detects moving areas via subtracting the present image from the reference one in a pixel-by-pixel basis. Although this algorithm roughly seems very similar to TD algorithm, Background Subtraction detects the background changes rather than moving objects as in TD. Conversely, it is straightforward and uncomplicated in achievement, and more accurate than a TD algorithm. The main drawback is its sensitivity to the external environment changes such as light or weather.

\subsubsection{Gaussian Mixture Models}

In 1999, Stauffer and Grimson [28], introduced this algorithm. It is based on a function of Gaussian probability density for evaluating the pixel intensity value. It determines the difference between the intensity value of the current pixel and the aggregate average of the previous values. Thus, it saves an aggregate average of the current pixel values. Now, if the difference between the value of the present pixel and the aggregate pixel is less than the product of the standard deviation with a constant value, then it is identified as a background; else, it is identified as foreground. This algorithm performs well with brightness levels, but it does not deal with the multi-model background.

\subsection{Analysis of Variance (ANOVA)}

The need of evidence for supporting an approach is an essential task to make a decision with high confidence and reliability. Thus, the ANOVA concept should take into consideration. For instance, a common approach for figuring out a dependable accuracy of an algorithm is via analyzing its performance. It is possible to use a statistical method to compare, for example, three different datasets in order to evaluate the difference between these datasets. ANOVA is the method that compares datasets based on their means. Consequently, the use of ANOVA includes some crucial assumptions, which involve independence of case, normality, and homogeneity. However, ANOVA is the best technique if the resultant data achieves these assumptions [29].

On the other hand, there are three types of ANOVA, which are one-way, two-way, and K-way analysis. All these types use at least three groups or sets for comparison. The difference is the number of factor (so-called independent) variables that the ANOVA type is based on. For instance, one factor variable is for one-way type, two factor variables are for two-way type, and three or more are for K-way type. Note that it is absolutely usual for the factors to be called "independent variables". Furthermore, it is worth of importance to note that the K-way ANOVA is commonly called Multi-variate ANOVA (abbreviated as MANOVA). For instance, if three workers represent three groups, then the factor variable will be the working hours for one-way, the working hours and the working condition for two-way, and the working hours, the working condition, and the total rest time for K-way [29].

\subsubsection{K-way ANOVA (MANOVA)}

The purpose of MANOVA is to observe the change of the dependent variable via employing the independent variable. In other words, if interaction validity is there among three or more independent variables on a dependent variable, then K-way comes into play. For example, it is interested to examine the impact of different textbooks on the student score in science and mathematics. Then, the enhancements in science and mathematics means that we have two dependent variables, hence MANOVA is suitable and applicable. Note that MANOVA gives a multivariate F-value while ANOVA gives univariate (single) Fvalue. The F-value is an output result generated by several statistical tests. ANOVA used the F-value to obtain the test status, which is either statistically significant or not. It is computed by dividing two mean squares. Basically, F-value is determined as [30, 31]:

F-value $=$ explained variance $/$ unexplained variance

"The following procedure describes how the $\mathrm{F}$ value is calculated:

1. Calculate the overall mean for the continuous field.

2. Calculate the mean square for the categorical field (the explained variance).

a. Calculate the sum of squares for the categorical field.

i. For each category, subtract overall mean from the category's mean.

ii. Take the square of each of these results and add them together.

b. Divide the sum of squares for the categorical field by the appropriate degrees of freedom.

3. Calculate the mean square for the error source (the unexplained variance).

a. Calculate the sum of squares for the error source.

i. Within each category, subtract the category's mean from each record value.

ii. Take the square of each difference and add them together. 
b. Divide the sum of square for the error source by the appropriate degrees of freedom.

4. Divide the mean square for the categorical field by the mean square for the error source. In other words, calculate the ratio of explained variance to unexplained variance. This is the F value."

However, MANOVA examines the dependent variables via generating a novel, synthetic, dependent variables in order to enlarge the group differences. These generated variables are direct groupings of the measured dependent variables.

Understanding the MANOVA results is so simple. If statistically significant is the multivariate F-value status, then something is significant. In other words, getting significant result means the need for looking at each univariate f-value (individual component) to obtain which dependent variable influenced to the significant result.

Conversely, this paper answers the question "how to perform a three-way ANOVA utilizing SPSS statistics?", in addition to clarify and show up the results from this test. In contrast, getting a valid results needs that the group data must meet some assumptions, similar to ANOVA, such as independence of case, normality, and homogeneity. These assumptions are [31]:

1) The dependent variable must be continuous (ratio or interval).

2) The three independent variables must each has at least two independent groups.

3) There is independence of observations in each group, as well as between the groups themselves.

4) There is not significant (unusual) data within each group.

5) The dependent variable must be normal distribution over each group combination.

6) Each group combination of the three independent variables must be homogeneity of variances.

In addition, the following MANOVA concepts and key terms are computed as follows [31]:

a) Sum of Squares between Groups (BSS): First, the individual means of the group is calculated. Next, get the deviation from the individual mean of each group. Finally, square the individual group and determine the sum of all groups.

b) Sum of Squares within a group (WSS): For all groups, compute the grand mean. Next, get the deviation from the individual group. Finally, square the deviation and determine the sum of all groups.

c) F-ratio: It is computed by dividing the sum of the squares between groups by the sum of the squares within a group.

d) Degree of Freedom: Subtract one from the number of group to calculate the degree of freedom between the sums of the squares group (i.e. $\mathrm{df}=(\mathrm{g}-1)$, where $\mathrm{df}$ is the degree of freedom and $\mathrm{g}$ is the number of groups). In addition, subtract the number of groups from the total observation to get the sum of the square within the group's degree of freedom (i.e. $\mathrm{df}=(\mathrm{N}-\mathrm{g})$, where $\mathrm{N}$ is the total sample size).

In this study, the used model consists of three detecting algorithms. Each algorithm has three groups. In addition, independent variables, which are false recognition, misrecognition, and camera's auto white-balancing compensation. The mathematical representation of the model is:

$$
Y_{i j k m}=\mu+A_{i}+B_{j}+C_{k}+A B_{i j}+A C_{i k}+B C_{j k}+A B C_{i j k}+\varepsilon_{m(i j k)}
$$

Where $A, B$, and $C$ are the independent variables, $i=j=1,2$, and $3, k=1$ and 2 .

\section{RESULTS}

As mentioned earlier, three detecting video surveillance algorithms, which are Temporal Differencing, Background Subtraction, and Gaussian Mixture Models, are employed in this study, due to its highest accuracy among other algorithms. The selection of which of them is the best one is a harmful task. Although accuracy is the best parameter for differentiating between them, but unfortunately it does not work due to two reasons. The first is all algorithms have accuracy close to each other and no significant difference between them is there. The second reason is that these algorithms are developed from different sources, i.e. each has its own datasets, and thus the accuracy parameter becomes a good parameter rather than the best.

The solution for the second reason is to apply the same datasets for all these algorithms under consideration. Three different datasets (groups) are applied to these algorithms, where each dataset is a sample video. All these videos have a frame size of $(120 \mathrm{x} 160)$ pixels, but with different duration. The statistical analysis depends on (MATLAB 2018b) outputs of the information that accurately describe the recognition process of the image system. The results are shown in Table 1. At the first glance, there is no significant difference in terms of overall object valid occurrence (OVO) among these algorithms. The maximum difference of less than $2 \%$ (in OVO) means that their accuracy are too close to each other. Therefore, the need for further analysis of the other variables (in the Table 1) in depth is necessary.

Initially, considering the variable "Total Fragmentation", clearly, the Temporal Differencing algorithm is the worst in object fragmentation. It detected thirteen objects while on the ground-truth is only 
ten. In addition, it benefited from white-balance compensation (dropping image mean value) and the fragmentation ratio became 1.2 instead of 1.3. Furthermore, the Temporal Differencing algorithm suffers from a severe deficiency, it is sensitive to object speed, and it enlarges fast objects, which gives them a chance for splitting. In contrast, the Background algorithm has a slightly better total fragmentation ratio (1.1) which means that it splits only one of the ten objects into two. In addition, the algorithm does not benefit from white-balance compensation, whereas the detected objects are eleven with or without compensation. This can be explained as it is not sensitive to object speed, and hence, there is no chance for more objects splitting. Finally, the Gaussian Mixture Models algorithm gives the best total fragmentation ratio. It is perfect when used with white-balance compensation.

However, when looking to the variable "False Recognition Ratio", the Background algorithm is the perfect one. It did not falsely recognize any object. The other two algorithms are rather inaccurate, and they did mistakenly recognize the wanted object. In contrast, considering the variable "True Recognition Ratio" in Table (1) shows that the Background algorithm is again the best in detecting true wanted object's occurrences. Moreover, the variable "Misrecognition Ratio" shows that the Background algorithm is the best. It lost only one out of thirteen of object's occurrences along its track.

On the other hand, all tested algorithms are benefited from white-balance compensation, and they become more efficient and powerful. Background algorithm is the best choice when a system with no false alarms is required. Furthermore, this algorithm is the fastest among the others (as in Table 4.1), which allows high frames per second (fps) processing when fast target objects are to be tracked.

Table 1. Statistically Performance Analysis of Three Detection Algorithms

\begin{tabular}{|c|c|c|c|c|c|c|}
\hline Detection algorithm & \multicolumn{2}{|c|}{$\begin{array}{l}\text { Temporal Differencing } \\
F(n)-F(n-1)\end{array}$} & \multicolumn{2}{|c|}{$\begin{array}{l}\text { Fixed Background } \\
\text { Subtraction }\end{array}$} & \multicolumn{2}{|c|}{$\begin{array}{c}\text { Gaussian Mixture } \\
\text { Models }\end{array}$} \\
\hline \multirow{5}{*}{ Object Valid Occurrences (OVO)* } & \multicolumn{2}{|c|}{11} & \multicolumn{2}{|c|}{13} & \multicolumn{2}{|c|}{12} \\
\hline & \multicolumn{2}{|c|}{15} & \multicolumn{2}{|c|}{17} & \multicolumn{2}{|c|}{16} \\
\hline & \multicolumn{2}{|c|}{44} & \multicolumn{2}{|c|}{46} & \multicolumn{2}{|c|}{45} \\
\hline & \multicolumn{6}{|c|}{ Using Camera's Auto White-Balancing Compensation } \\
\hline & Yes & No & Yes & No & Yes & No \\
\hline Total Fragmentation Ratio & 1.2 & 1.3 & 1.1 & 1.1 & 1.0 & 1.1 \\
\hline$=$ total detected objects $\div(10$, the total ground - & 1.6 & 1.8 & 1.5 & 1.5 & 1.4 & 1.4 \\
\hline truth video objects**) & 5.6 & 5.7 & 5.4 & 5.4 & 5.3 & 5.4 \\
\hline False Recognition Ratio & 0.18 & 0.27 & 0.00 & 0.00 & 0.16 & 0.25 \\
\hline \multirow{3}{*}{$\begin{array}{c}=\text { False Recognized Occurrences }(\mathrm{FRO}) \div \\
(\mathrm{OVO}) *\end{array}$} & 0.13 & 0.2 & 0.00 & 0.00 & 0.12 & 0.18 \\
\hline & 0.15 & 0.18 & 0.00 & 0.00 & 0.15 & 0.17 \\
\hline & $2 / 11$ & $3 / 11$ & $0 / 13$ & $0 / 13$ & $2 / 12$ & $3 / 12$ \\
\hline \multirow[t]{2}{*}{$(\mathrm{FRO}) /(\mathrm{OVO}) *$} & $2 / 15$ & $3 / 15$ & $0 / 17$ & $0 / 17$ & $2 / 16$ & $3 / 16$ \\
\hline & $7 / 44$ & $8 / 44$ & $0 / 46$ & $0 / 46$ & $7 / 45$ & $8 / 45$ \\
\hline Misrecognition Ratio & 0.09 & 0.00 & 0.07 & 0.23 & 0.08 & 0.08 \\
\hline \multirow{3}{*}{$=$ Un-Recognized Occurrences $(\mathrm{URO}) \div(\mathrm{OVO})$} & 0.06 & 0.00 & 0.05 & 0.29 & 0.06 & 0.06 \\
\hline & 0.02 & 0.00 & 0.02 & 0.06 & 0.02 & 0.02 \\
\hline & $1 / 11$ & $0 / 11$ & $1 / 13$ & $3 / 13$ & $1 / 12$ & $1 / 12$ \\
\hline \multirow[t]{2}{*}{$(\mathrm{URO}) /(\mathrm{OVO}) *$} & $1 / 15$ & $0 / 15$ & $1 / 17$ & $5 / 17$ & $1 / 16$ & $1 / 16$ \\
\hline & $1 / 44$ & $0 / 44$ & $1 / 46$ & $3 / 46$ & $1 / 45$ & $1 / 45$ \\
\hline True Recognition Ratio & 0.90 & 1.00 & 0.92 & 0.76 & 0.91 & 0.91 \\
\hline \multirow{3}{*}{$\begin{array}{c}=\text { True Recognized Occurrences }(\mathrm{TRO}) \div \\
(\mathrm{OVO}) *\end{array}$} & 0.93 & 1.00 & 0.94 & 0.70 & 0.93 & 0.93 \\
\hline & 0.97 & 1.00 & 0.97 & 0.93 & 0.97 & 0.97 \\
\hline & $10 / 11$ & $11 / 11$ & $12 / 13$ & $10 / 13$ & $11 / 12$ & $11 / 12$ \\
\hline \multirow[t]{2}{*}{$(\mathrm{TRO}) /(\mathrm{OVO}) *$} & $14 / 15$ & $15 / 15$ & $16 / 17$ & $12 / 17$ & $15 / 16$ & $15 / 16$ \\
\hline & $43 / 44$ & $44 / 44$ & $45 / 46$ & $43 / 46$ & $44 / 45$ & $44 / 45$ \\
\hline
\end{tabular}

* Object Valid Occurrences; object occurrence is considered valid by foreground detector when object's boundaries are fully occupied inside the region of interest.

** A ground-truth object is what the human eye decides as object.

In summary, no algorithm is selected as the perfect or suitable for all applications. Therefore, going further with these variables using another approach, rather than the accuracy analysis. The proposed approach, we are looking for, is to give us an evidence for making a reliable and confident decision. Hence, the K-way ANOVA test comes into play using SPSS program.

Initially, to get a valid result, the six assumptions, mentioned earlier, must be satisfied. The data sets must meet assumptions 1, 2, and 3 before applying the K-way ANOVA, while the results must satisfy the assumptions 4, 5, and 6 . In contrast, defining the dependent and independent variables must be clarified well, early. However, the independent variables are:

F1: the first factor represents the three algorithms (TD, BS, and GMM) under consideration.

F2: the second factor represents three parameters (OVO, FRO, and URO). 
F3: the last factor represents the compensation of the camera's auto-white balancing (Yes or No).

Table 2 shows the K-way ANOVA output using SPSS program. Understanding the results is so simple. If statistically significant is the multivariate F-value status, then something is significant. In other words, getting significant result means the need for looking at each univariate F-value (individual component) to obtain which dependent variable influenced to the significant result. In Table 2, F-value is statistically significant only with the second factor F2. In addition, the third factor has not a significant effect, as well as, the first factor. Therefore, it can be concluded that the selection of which algorithm to be used based on accuracy is so significant, instead, looking for an algorithm with lowest false recognition ratio or unrecognized ratio. Thus, the K-way ANOVA proofs the carried out results of Table 1.

Table 2. The output of 3-way ANOVA using SPSS

\begin{tabular}{|c|c|c|c|c|c|}
\hline \multicolumn{6}{|c|}{ Tests of Between-Subjects Effects } \\
\hline Source & $\begin{array}{c}\text { Type III Sum } \\
\text { of Squares }\end{array}$ & $\mathrm{df}$ & Mean Square & $\mathrm{F}$ & Sig. \\
\hline Corrected Model & $6449.508^{\mathrm{a}}$ & 17 & 379.383 & 3.450 & .001 \\
\hline Intercept & 2949.770 & 1 & 2949.770 & 26.825 & .000 \\
\hline $\mathrm{F} 1$ & 12.986 & 2 & 6.493 & .059 & .943 \\
\hline F2 & 6368.010 & 3 & 2122.670 & 19.303 & .000 \\
\hline F3 & .471 & 1 & .471 & .004 & .948 \\
\hline $\mathrm{F} 1{ }^{*} \mathrm{~F} 2$ & 68.483 & 3 & 22.828 & .208 & .890 \\
\hline $\mathrm{F} 1{ }^{*} \mathrm{~F} 3$ & .284 & 2 & .142 & .001 & .999 \\
\hline $\mathrm{F} 2{ }^{*} \mathrm{~F} 3$ & 1.334 & 3 & .445 & .004 & 1.000 \\
\hline $\mathrm{F} 1{ }^{*} \mathrm{~F} 2{ }^{*} \mathrm{~F} 3$ & .747 & 3 & .249 & .002 & 1.000 \\
\hline Error & 3958.703 & 36 & 109.964 & & \\
\hline Total & 14840.181 & 54 & & & \\
\hline Corrected Total & 10408.210 & 53 & & & \\
\hline
\end{tabular}

\section{CONCLUSIONS}

This paper presented further analysis of the accuracy. The results proofed that: No algorithm is selected as the perfect or suitable for all applications; all tested algorithms are benefited from white-balance compensation, and they become more efficient and powerful; false recognition ratio and unrecognized ratio parameters have a significant impact; in addition, to get a significant result means the need for looking at each univariate F-value to obtain which dependent variable influenced to the significant result; the K-way ANOVA test with SPSS program gave a reliable and confident decision.

\section{REFRENCES}

[1] Carlos Cuevas, and NarcisoGarcía, "Improved Background Modeling For Real-time Spatio-temporal Nonparametric Moving Object Detection Strategies", Elsevier, Image and Vision Computing, 31, pag. 616-630, 2013.

[2] Pritam Das, RanjitGhoshal, Dipak Kumar Kole, and Rabindranath Ghosh, "Measurement of Displacement and Velocity of a Moving Object from Real Time Video", International Journal of Computer Applications, (0975-8887) Volume 49-No. 13, July 2012.

[3] Dashan Gao and Jie Zhou, "Adaptive Background Estimation for Real-time Traffic Monitoring", IEEE Intelligent Transportation Systems Conference Proceedings - Oakland, USA-August 25-29, 2001.

[4] Deng, Jianxin. "Architecture design of the vehicle tracking system based on RFID." TELKOMNIKA (Indonesian Journal of Electrical Engineering), 11.6 (2013): 2997-3004.

[5] Humaidi, Amjad J., Mohammed Abdulraheem Fadhel, and Ahmed R. Ajel. "Lane detection system for day vision using altera DE2." TELKOMNIKA (Indonesian Journal of Electrical Engineering), 17.1 (2019): 349-361.

[6] PlamenAngelov, RaminRamezani and Xiaowei Zhou, Student Member, "Autonomous Novelty Detection and Object Tracking in Video Streams using Evolving Clustering and Takagi-Sugeno Type Neuro-Fuzzy System", IEEE, International Joint Conference on Neural Networks 2008.

[7] G. Prabhakar and B. Ramasubramanian, "An Efficient Approach for Real Time Tracking of Intruder and Abandoned Object in Video Surveillance System", International Journal of Computer Applications, (0975-8887) Volume 54-No.17, September 2012.

[8] Kamal Sehairi, Fatima Chouireb, Jean Meunier, "Comparative study of motion detection methods for video surveillance systems,” J. Electron. Imaging, 26(2), 023025 doi: 10.1117/1.JEI.26.2.02302, 2017. 
[9] Isabel Martins, Pedro Carvalho, Luís Corte- RealJosé \& Luis Alba-Castro, "BMOG: Boosted Gaussian Mixture Model with Controlled Complexity," Iberian Conference on Pattern Recognition and Image Analysis, vol.547, No 2, pp. 50-57, August 2017.

[10] Henry Schneiderman and Takeo Kanade, "A Statistical Method for 3D Object Detection Applied to Faces and Cars", IEEE, 2001.

[11] R. Cucchiara, P. Onfiani, A. Prati, N. Scarabottolo, "Segmentation of Moving Objects at Frame Rate: A Dedicated Hardware Solution", Proceedings of 7th IEE Conference on Image Processing and its Applications, 1999.

[12] Fadhel, Mohammed Abdulraheem, Omran Al-Shamaa, and Bahaa Husain Taher. "Real-Time detection and tracking moving vehicles for video surveillance systems using FPGA." International Journal of Engineering \& Technology, 7.2.31 (2018): 117-121.

[13] Hassan, Inaam Rikan and Mohammed Abdulraheem Fadhel. "Tracking Vehicles in Urban Smart City Based Xilinx Platform.”, Journal of Theoretical and Applied Information Technology, (2019).

[14] Albiol, Antonio, Maria Julia Silla, Alberto Albiol, and Jose Manuel Mossi. "Video analysis using corner motion statistics." In IEEE International Workshop on Performance Evaluation of Tracking and Surveillance, pp. 31-38. 2009.

[15] Liu, Chang, Guijin Wang, Wenxin Ning, Xinggang Lin, Liang Li, and Zhou Liu. "Anomaly detection in surveillance video using motion direction statistics." In 2010 IEEE International Conference on Image Processing, pp. 717-720. IEEE, 2010.

[16] Zhou, Wenjun, Shun'ichi Kaneko, Manabu Hashimoto, Yutaka Satoh, and Dong Liang. "Co-occurrence Background Model with Hypothesis on Degradation Modification for Robust Object Detection." In VISIGRAPP (5: VISAPP), pp. 266-273. 2018.

[17] Slawomir Bak, Marco San Biagio, Ratnesh Kumar, Vittorio Murino, François Bremond. Exploiting Feature Correlations by Brownian Statistics for People Detection and Recognition. IEEE transactions on systems, man, and cybernetics, Institute of Electrical and Electronics Engineers (IEEE), 2016. (hal-01850064).

[18] Warne, Russell T. "A Primer on Multivariate Analysis of Variance (MANOVA) for Behavioral Scientists." Practical Assessment, Research \& Evaluation, 19 (2014).

[19] Konietschke, Frank, et al. "Parametric and nonparametric bootstrap methods for general MANOVA." Journal of Multivariate Analysis, 140 (2015): 291-301.

[20] Engel, J., et al. "Regularized MANOVA (rMANOVA) in untargeted metabolomics." Analytica chimica acta, 899 (2015): 1-12.

[21] Asiltürk, Ilhan, Süleyman Neşeli, and Mehmet Alper Ince. "Optimisation of parameters affecting surface roughness of Co28Cr6Mo medical material during CNC lathe machining by using the Taguchi and RSM methods." Measurement, 78 (2016): 120-128.

[22] Alzubaidi, Laith, Mohammed A. Fadhel, Sameer R. Oleiwi, Omran Al-Shamma, and Jinglan Zhang. "DFU_QUTNet: diabetic foot ulcer classification using novel deep convolutional neural network." Multimedia Tools and Applications, (2019): 1-23.

[23] Alzubaidi, Laith, Omran Al-Shamma, Mohammed A. Fadhel, Laith Farhan, and Jinglan Zhang. "Classification of Red Blood Cells in Sickle Cell Anemia Using Deep Convolutional Neural Network." In International Conference on Intelligent Systems Design and Applications, pp. 550-559. Springer, Cham, 2018.

[24] Al-Shamma, Omran, Mohammed Abdulraheem Fadhel, Rabab Alaa Hameed, Laith Alzubaidi, and Jinglan Zhang. "Boosting Convolutional Neural Networks Performance Based on FPGA Accelerator", In International Conference on Intelligent Systems Design and Applications, pp. 509-517. Springer, Cham, 2018.

[25] Pan, Chun, Zhiguo Yan, Xiaoming Xu, Mingxia Sun, Jie Shao, and Di Wu. "Vehicle logo recognition based on deep learning architecture in video surveillance for intelligent traffic system", (2013): 132-135.

[26] Mahamuni P.D, R.P Patil and H.S.Thakar, "Moving Object Detection Using Background Subtraction Algorithm using simulink" in IJERT, Volume 3, Issue 6, 2014.

[27] Asim R. Aldhheri and Eran A. Edirisinghe, "Detection and classification of a moving object in a video stream" in ACIT, 2014.

[28] Santoyo-Morales, Juana E., and Rogelio Hasimoto-Beltran. "Video background subtraction in complex environments." Journal of applied research and technology, 12.3 (2014): 527-537.

[29] Cardinal, Rudolf N., and Michael RF Aitken. "ANOVA for the behavioral sciences researcher", Psychology Press, 2013.

[30] Boisgontier, Matthieu P., and Boris Cheval. "The anova to mixed model transition." Neuroscience \& Biobehavioral Reviews, 68 (2016): 1004-1005.

[31] Chatfield, Chris, "Introduction to multivariate analysis" Routledge, 2018.

\footnotetext{
Statistical accuracy analysis of different detecting algorithms for surveillance system... (Hassan Al-Yassin)
} 


\section{BIOGRAPHIES OF AUTHORS}

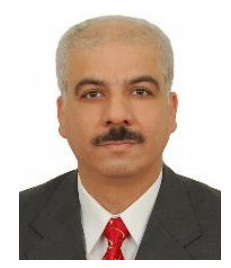

Hassan Al-Yassin is a lecturer of statistics since 1992. Worked as a statistical consultant for distribution of students in Iraqi universities (1993-1996, Ministry of Higher education and scientific research). Also worked at the Cultural Attaché in Moscow for the period 2006-2010. Worked in the Department of Missions and Cultural Relations with International Educational Organizations (2011 - 2016). Finished M.Sc. in Statistics (1992 Al-Mustansiriyah University). Ph.D. in economics (2005 Moscow State University of Economics, Statistics, and Informatics (MESI).2016 till now, working in some researches in applied statistics, biostatistics, oil statistics, IT statistics.

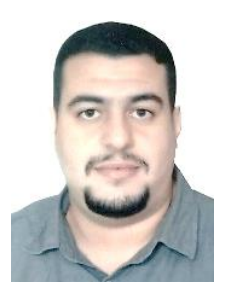

Jaafar I. Mousa currently works as a principle lecturer in Israa University College, since 2017. $\mathrm{He}$ received his BSc. And MSc degree in applied mathematics, University of Technolgy, Baghdad, Iraq, 2014 and 2016, respectively. His interest in Fuzzy theory applications and applied mathematics in engineering.

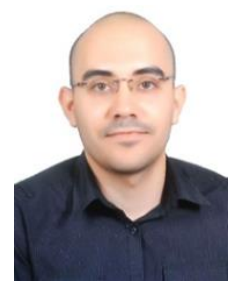

Mohammed A. Fadhel works at the University of Information Technology and Communications, Business informatics collage Baghdad, Iraq. He gets MSc. from University of Technology/Control and Systems Eng., Baghdad, IRAQ 2015. Mohammed interests in FPGA, medical diagnosis techniques, and real-time survallance systems, artificial intelligence.

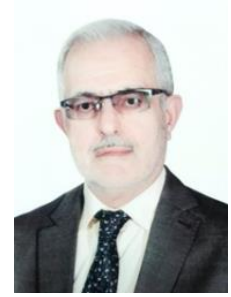

Omran Al-Shamma currently works at the Postgraduate affairs, University of Information Technology and Communications, Baghdad, Iraq. He holds $\mathrm{PhD}$ from University of Hertfordshire, UK 2013. Omran interests in computer design software, medical diagnosis techniques, and preliminary aircraft design software, Real time survallace system.

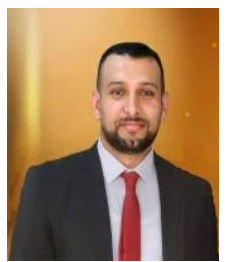

Laith Alzubaidi is currently a Ph.D. student at Queensland University of Technology / Faculty of Science and Engineering, Brisbane, Australia. He received his Master's degree in computer science from the University of Missouri/USA in 2016. In his Master's thesis, he worked on the detection and classification of breast cancer with deep learning. Laith broad research area falls in artificial intelligence and Internet of Things (IoT). In particular, his research interests include Deep Learning for medical applications and IoT. 